\title{
Understanding the interaction properties of an eco- friendly corrosion inhibitor on Zn (1 110$)$ surface: Comprehensive DFT-based MD simulation
}

\section{Atyeh Rahmanzadeh}

Islamic Azad University Medical Branch of Tehran

Mahyar Rezvani

Islamic Azad University, Arak

Masoud Darvish Ganji ( $\sim$ ganji_md@yahoo.com )

Qaemshahr Islamic Azad University

\section{Research Article}

Keywords: Coating, Green inhibitor, Corrosion, Surface, DFT, MD simulation

Posted Date: January 5th, 2021

DOl: https://doi.org/10.21203/rs.3.rs-135917/v1

License: (c) (i) This work is licensed under a Creative Commons Attribution 4.0 International License.

Read Full License 


\section{Abstract}

Regarding the deleterious effects of corrosion for a wide range of metals and alloys, many different techniques have been developed to protect the metals against corrosion. Utilizing organic inhibitors, especially those that contain heteroatoms and multiple bonds has been found an effective approach. In this research, the adsorption of a novel green inhibitor, Laurhydrazide N'-propan-3-one (LHP), on the Zn (110) surface was investigated using dispersion corrected DFT calculations. Interaction energy and electronic structures were calculated for different orientations of the inhibitor toward the Zn surface. The validity of calculated interaction energy has been verified by the MP2 level of theory. The AIM theory analysis revealed that LHP bound strongly to the $\mathrm{Zn}$ surface through its $\mathrm{O}$ active site and also its orientation affects greatly the interaction energy. Furthermore, diffusion of LHP through its $\mathrm{O}$ atoms active sites was observed with the state-of-the-art DFT-MD simulation during the simulation procedure that agrees well with the experiments for similar molecules adsorbed on the metal surfaces. The presented findings afford a vital insight into the interactive nature of adsorbed inhibitors on metallic surfaces and will help to develop advanced functional materials in coating technologies.

\section{Introduction}

In general, the first technical innovation and utilization of metallic zinc date back to the mid- $13^{\text {th }}$ century. Before this era, the major use of zinc ores was fabricating brass [1-3]. In recent decades, zinc and zincbased alloys have been broadly used in assorted industries such as die-casting, Nano-zinc oxide, hot-dip galvanizing, zinc chemicals, coating, wrought alloys, and electronics [4-11]. Zinc and its alloy are preferable anodic materials in various high-energy batteries including $\mathrm{Zn} /$ air, $\mathrm{Ni} / \mathrm{Zn}$, and $\mathrm{Ag} / \mathrm{Zn}$ batteries [12-16]. Furthermore, owing to the facile application, great metallurgical property, sacrificial behavior, and cost-effectivity, zinc has been considerably utilized in protecting carbon steel from corrosion [17-21]. Despite the fact that zinc has reasonable resistance against corrosion, distinct aggressive media such as aqueous/acidic $(\mathrm{pH}<6)$ /alkaline $(\mathrm{pH}>12.5)$ solutions, atmospheric ambient and the circumstances implemented to accelerate the experiments, for instance, cyclic and spray tests, can create detrimental corrosion effects on the surface of zinc [22-28]. One of the well-established approaches for the protection of diverse metals against devastating corrosions is using chemical interaction inhibitors [29-33]. According to the literature, organic inhibitors, particularly those contain heteroatoms, namely, P, S, O, and $\mathrm{N}$, and also multiple bonds, for example, $\mathrm{C}=\mathrm{C}, \mathrm{N}=\mathrm{N}, \mathrm{C}=\mathrm{O}, \mathrm{C} \equiv \mathrm{N}$, etc., are able to diminish the probability of corrosion and also protect the metal surface effectively [34-37]. The distinguished performance of this class of organic inhibitors could be ascribed to the adsorption of monomers on the surface of metals via aromatic rings, multiple bonds, and electron donor heteroatoms [38-40]. It is worth to point that the simultaneous presence of -bonding and heteroatoms within inhibitor structure can improve the protection ability of applied inhibitor through strong chemical or physical adsorption and forming a mono-/multilayer protective film on the metal surface [41-43]. A great deal of investigations has been allocated to the corrosion inhibition behavior of amino acids for different metals and alloys [44-48]. Amino acids are a group of fundamental biomolecules that play a key role in building blocks of elemental substances 
including medicine and nutrition [49-52]. The primary structure of amino acids is mainly constructed from carboxyl $(-\mathrm{COOH})$ and amino $\left(-\mathrm{NH}_{2}\right)$ groups $[53,54]$. Amino acids are also known as inexpensive, ecoenvironmentally friendly, simply producible corrosion inhibitor [55-57]. Badawy et al. studied about corrosion inhibition behavior of various amino acids to protect the surface of $\mathrm{Cu}-\mathrm{Ni}$ alloys in neutral chloride media [58]. For this purpose, they utilized electrochemical technics and they found out that amongst several considered amino acids, cysteine exhibited the greatest inhibition efficiency. AshassiSorkhabi and coworkers by taking advantage of potentiodynamic polarization assessed the inhibitory effects of three different amino acids for steel corrosion in acidic solutions [59]. They realized that the compounds can act as efficient inhibitors. Kaya et al. attempted to protect the surface of $\mathrm{Cu}(111)$ by using quantum chemical computations and Molecular Dynamic (MD) implementing Monte Carlo (MC) method [60]. The obtained results were compared to experimental counterparts and it was revealed that there is a favorable correlation between them. In the present research, the formation of a protective molecular layer of Laurhydrazide N'-propan-3-one (LHP) as a diamino acid on Zn (110) is comprehensively studied. The brilliant protective behavior of this environmental-friendly inhibitor has been already confirmed by a group of researchers using various experimental techniques [61]. Herein, for the first time, the interactive nature and adsorption mechanism of the formed protective LHP layer on the surface of Zn (110) will be studied. For this aim, Density Functional Theory (DFT) was executed to conduct a precise investigation of the electronic and structural features of interacting molecular systems. Moreover, DFT-based molecular dynamic (MD) simulation was employed to model realistic molecular systems at ambient conditions. Our obtained results illuminate that LHP adsorbed strongly on the $\mathrm{Zn}$ surface and different orientations of LHP toward the $\mathrm{Zn}$ surface can greatly influence the formation of a protective layer on the surface. Meanwhile, DFT-MD simulation showed that LHP diffused on the Zn surface at environmental conditions.

\section{Computational Procedures}

In order to scrutinize the possible formation of the protective layer and adsorption configurations of LHP on the surface of Zn, ab initio DFT approach was carried out with SIESTA (Spanish Initiative for Electronic Simulations with Thousands of Atoms) simulation computer program [62, 63]. All computational procedures with the SIESTA code were executed in a platform of generalized gradient approximation (GGA) considering Perdew-Burke-Ernzerhof (PBE) exchange-correlation functional (GGA-PBE) [64] and Troullier-Martins pseudopotential for the description of exchange-correlation potential [65-67]. The SIESTA code is able to make intelligible the atomic cores of valence electrons by using localized numerical basis sets, periodic boundary conditions (PBCs), and norm-conserving nonlocal pseudopotentials. Furthermore, the Norm-conserving pseudopotentials were all meticulously assembled to define the interaction of valance electrons containing atomic cores $[68,69]$. In addition, for the valance wave function and also double- $\zeta$ basis sets, the localized atomic orbitals were implemented and cutoff energy of 200 Ry was considered for the studied systems. 
Moreover, DFT-MD simulation was carried out too by utilizing SIESTA code and employing the dynamics approximation of Born-Oppenheimer to simulate the molecular systems. The interaction amongst ions and electrons was explained through the GGA-PBE model of the theory. In order to solve Newton's equations of motion, the Verlet algorithm was utilized. The NVT ensemble was fulfilled by considering a Nosé-Hoover thermostat to retain the temperature at $300 \mathrm{~K}$.

Furthermore, first-principle computations were carried out by resorting to the DFT approach utilizing OpenMX code which stands for Open Source Package for Material eXplore. The OpenMX code is mainly founded on the norm-conserving pseudopotentials and linear combination of pseudo-atomic orbital (LCPAO) basis function through the local density approximation (LDA) [70]. Further, the pseudo-atomic orbitals were used as a basis set. The electron cores were explained by Kleinman-Bylander Pseudopotentials, norm-conserving Morrison, and also the GGA considering the PBE functional was implemented for correlation and exchange terms $[67,71]$. In order to evaluate the charge density in real space, the cutoff in kinetic energy was regulated 200 Ry for the grid integration. In furtherance of structural relaxation, till the net forces reach to $1 \times 10^{-4}$ a.u, the rational function (RF) approach of quasinewton was performed. In this investigation, a supercell consisted of 110 zinc atoms is considered, and a periodic boundary condition (PBC) was enforced. The interaction energies $\left(E_{\text {int }}\right)$ of the adsorbate, namely, LHP and $\mathrm{Zn}$ sheet as the substrate were measured via the following equation:

$E_{\mathrm{int}}=E($ complex $)-\left[E_{(\text {sub })}+E_{(\text {ads })}\right]-\delta_{\text {BSSE }}(1)$

In this equation, $E_{\text {(complex), }} E_{\text {(ads) }}$ and $E_{\text {(sub) }}$ represent the total energies of the studied complexes, adsorbates, and substrates, respectively. Moreover, the impact of the Basis Set Superposition Error (BSSE) in the calculation of the interaction energy was taken into account through the counterpoise correction (CP) approach [73] ( $\delta_{\mathrm{BSSE}}$ term).

In all calculations, with both SIESTA and OpenMX codes, the long-range dispersion correction for nonbonding vdW interactions was considered investigated by the DFT-D2 method proposed by Grimme [72].

Moreover, the accuracy of the results achieved through the performed DFT-PBE method was evaluated by the second-order Møller-Plesset perturbation (MP2) level of theory. For this purpose, the triple- $\zeta$ valence plus polarization basis set without considering $f$ orbitals (def2-TZVP(-f)) for light atoms described by Ahlrichs was enforced for geometrical optimization [74]. Besides, the theoretical model of def2-TZVP was used for electronic structures and total energy. Since transition metal exists in our fundamental structure, effective core potential (ECP) for $Z n$ atoms was used $[75,76]$. The method of nucleus effective charge was applied for $\mathrm{Zn}$ atoms, too.

We also employed the theory of atoms in molecules (AIM) method to explore the interactive nature of interacting systems. Further details and discussions about the AIM theory can be found in the research literature [77-79]. To this object, the ORCA software was utilized to realize the wave-function engaged in 
the bonding survey at the DFT level of theory. Furthermore, the calculation of topological parameters and local features analysis of interacting molecules was performed by Multiwfn visualizer software [80].

\section{Results And Discussions}

In the first step, all considered structures including the Zinc-based substrate which is supposed to be coated with the selected inhibitor, namely LHP, were meticulously optimized using DFT-D calculations. A supercell of $26.23 \AA \times 10.50 \AA$ sheet containing two-atom-thickness and $110 \mathrm{Zn}$ atoms were considered. The optimized structures indicated that in the $\mathrm{Zn}$ sheet the bonding distance between two vicinal $\mathrm{Zn}$ atoms is about $2.627 \AA$ and the bond angle between two adjacent bonds was around $60^{\circ}$ (See Fig. 1a). Besides, the difference between the two considered parallel layers of the $\mathrm{Zn}$ sheet was about $3.013 \AA$, as depicted in the figure (side-view). In the case of the optimized structure of LHP, the bonding distances and dihedral torsion between $\mathrm{O}-\mathrm{C}-\mathrm{N}-\mathrm{H}$ were determined and presented in Fig. 1b. Our structural geometries data with DFT-D/PBE method for both considered systems has a desirable agreement with published reports [81-84].

\subsection{Adsorption of LHP on Zn (110) surface}

In order to investigate the adsorption behavior of LHP inhibitor on the Zn surface, two main configurations were considered. Regarding the various active sites of our selected inhibitor and metal surface, in the first configuration, LHP was located through one of its $\mathrm{O}$ atoms and the backbone of the inhibitor was perpendicular to the surface of the $Z n$ sheet. In this configuration, the 0 atom either can be located above a single $\mathrm{Zn}$ atom (top-site) or above three $\mathrm{Zn}$ atoms (hollow-site). For the second configuration, the inhibitor was positioned so that both $\mathrm{O}$ atoms were approached to the $\mathrm{Zn}$ atoms and its backbone was paralleled to the surface. Three modeled orientations are shown in Fig. 1c-e.

Following the full structural relaxation of the whole system, the most stable complex was ascertained by calculating the interaction energies with the BSSE correction for all considered configurations. The obtained data revealed that when LHP is parallel to the surface of the $\mathrm{Zn}$ sheet, the interaction energy is - $2.322 \mathrm{eV}$, while for other configurations, namely, LHP is perpendicular to the $\mathrm{Zn}$ surface the interaction energy is about $-1.144 \mathrm{eV}$ (Table 1). Therefore, the parallel orientation of LHP to the $\mathrm{Zn}$ surface results in better protection of the surface owing to the better coverage through strong adsorption between $\mathrm{O}$ and $\mathrm{Zn}$ atoms accompanied by the attraction of $-\mathrm{CH}_{2}$ groups over the $\mathrm{Zn}$ atoms. In the first configuration (perpendicular top-site), it was found that only a single $\mathrm{O}$ atom tends to interact with the $\mathrm{Zn}$ atom. The equilibrium distance of the $\mathrm{O}$ atom with the $\mathrm{Zn}$ atom is about $2.16 \AA$ and the equilibrium distance of $\mathrm{H}$ atoms with the surface is about $2.39 \AA$. The relaxed structure of this configuration is depicted in Fig. 2a. Moreover, for this configuration, the bonding distance of $\mathrm{Zn}-\mathrm{Zn}$ was about $2.695 \AA$. In the case of the second configuration (parallel), the equilibrium distance of $\mathrm{O}$ and $\mathrm{H}$ atoms with $\mathrm{Zn}$ atom after relaxation were about 2.341 and $2.526 \AA$, respectively (Fig. 2b). The bonding distance of $Z n-Z n$ near the 0 atom was $2.786 \AA$. Meanwhile, the calculated $Z n$... 0 distances was found to be almost larger than the sum of the covalent radii of $\mathrm{O}$ and $\mathrm{Zn}$ atoms (about $1.88 \AA$ ) and also seems to be rather larger than the reported 
covalent bond lengths for $\mathrm{Zn}-\mathrm{O}(\sim 1.9 \AA)$ reported in the literature [85-88]. Accordingly, this bond is not likely to be typical of a net covalent bond and needs further clarification by specialized analysis.

Table 1

Interaction energy $\left(E_{\text {int }}\right)$, charge transfer, and dispersion forces of considered LHP-Zn systems.

\begin{tabular}{|llll|}
\hline System & $\mathrm{E}_{\mathrm{int}}(\mathrm{eV})$ & $\mathrm{Q}_{\mathrm{T}}(\boldsymbol{e})$ Mulliken & Dispersion forces $(\mathrm{eV})$ \\
\hline LHP/Zn (Parallel) & -2.322 & 0.253 & -31.935 \\
\hline LHP/Zn (Perpendicular-Top) & -1.144 & 0.282 & -30.662 \\
\hline
\end{tabular}

The charge transfer analysis was performed through the Mulliken method and the attained data are presented in Table 1. It was found that when LHP is located in parallel to the $\mathrm{Zn}$ surface, a notable amount of electron (about $0.282 \mathrm{e}$ ) was transferred from LHP to the Zn surface. For the perpendicular orientation of LHP on the surface, the amount of $0.253 \mathrm{e}$ was transferred. The aforementioned amount of interaction energy, bonding distance, and electron transfer between the substrate and LHP, all declare the chemisorption nature of interaction [89-94].

To further elucidate the interactive nature of adsorbed inhibitor the long-range dispersion forces that play a significant role in the stability of such molecular systems $[67,68]$ was investigated. The exact description of non-local dispersion forces has been discerned as a major challenge for molecular systems due to the required great degree of electron correlations to be accounted for. Thanks to unparalleled endeavors by Grimme, it is a reasonable route to supplement empirical corrections to the energy of Kohn-Sham and take account of the dispersion forces for quantum mechanics methods. Our obtained PBE-D results indicate that adsorbed LHP molecule on the Zn surface for energetically configuration experience dispersion forces of $-31.935 \mathrm{eV}$. However, these forces for perpendicular orientation were estimated to be $-30.661 \mathrm{eV}$ as the consequence of the characteristic differences amongst the configuration of adsorbed molecules. From the obtained values for dispersion interactions, it was found that these forces play a significant role in LHP adsorption onto the $\mathrm{Zn}$ surface. In other words, it is obvious that dispersion forces make a strong impact on the adsorption and stability of such inhibitors on the metal surface.

We next evaluate the validity of the employed DFT-D2/PBE method in explaining the interaction of LHP with the surface of $Z n$ through benchmark calculation using the second-order Møller-Plesset perturbation (MP2) level of theory. In this regard, a cluster of $\mathrm{Zn}$ atoms consisted of seven $\mathrm{Zn}$ atoms was considered (Fig. 2c) and the corresponding interaction energy of the LHP-Zn system was calculated. First, we have performed full structural relaxation of the modeled cluster system and then the interaction energy was calculated with the BSSE correction through both above-mentioned methods. It was found the $E_{\text {int }}$ about $-0.453 \mathrm{eV}$ according to the data attained via DFT-D2/PBE method whilst the interaction energy through the MP2 method was calculated about $-0.317 \mathrm{eV}$. We found that the MP2 outcome 
confirms the PBE counterpart and both interaction energies for this configuration were in suitable agreement.

\subsection{Interactive nature of LHP/Zn system}

It was found from interaction energies with DFT-D calculations that the interactive nature of LHP with Zn surface is strong and estimated to be typical for the chemisorption. However, the type of interactions should be further evaluated to precisely clarify the interaction properties of the system under investigation. To this aim, the total electron density plots of the LHP-Zn system (cluster model) were calculated within the MP2 method and demonstrated in Fig. 2d. It can be observed from the obtained maps that there is a small charge accumulation in the interacting area between $\mathrm{O}$ and $\mathrm{Zn}$ atoms. This small electron density in the LHP-Zn interface can be attributed to a minor covalent bonding between adsorbed molecules on the surface which needs to be evaluated by further analysis such as the AIM theory scheme.

We next give deeper insight into the interactive nature of involved bonds in the complex through the AIM analysis fulfilled by the DFT method for the cluster system. The electronic charge density $(\rho(r))$ as well as Laplacian $\left(\nabla^{2} \rho(r)\right)$ and energy densities $H(r)$ were calculated and reported in Table 2. Also, the bond critical points (BCPs) for the relaxed structure of the LHP-Zn cluster system were estimated and represented in Fig. 2e. The data obtained for BCP for the bonding region of $\mathrm{O}$ and $\mathrm{Zn}$ atoms with the $\nabla^{2} \rho$ value of 0.223 reveals a solid charge depletion at the resultant critical point. The calculated $H(r)$ with a negative value of -0.005 a.u indicates the existence of an attractive interaction between involved entities ( $\mathrm{n}$ and $\mathrm{O}$ atoms). It was predictable that positive and negative values respectively for $\nabla^{2} \rho$ and $\mathrm{H}(\mathrm{r})$ indicate a strong polar attraction accompanied by a partially covalent bond between the neighbor nuclei. For comparison, the BCPs have been estimated for $\mathrm{Zn}-\mathrm{Zn}$ bond, and the data are listed in the table. As it was found, similar trends were observed for $\nabla^{2} \rho$ and $H(r)$ values that demonstrate the same type of bonding between $\mathrm{Zn}$...Zn atoms in the metal system (highly polar/partly covalent). Furthermore, the proportion of $\mathrm{G}(\mathrm{r}) / \mathrm{V}(\mathrm{r})$ that categorized in three levels $(0.1-0.5$ : covalent bond, $0.5-1$ : polar covalent bond and > 1: electrostatic bond) reveals that both $\mathrm{Zn}$... 0 and $\mathrm{Zn}$...Zn bonds are classified as a polar covalent bond with $\mathrm{G}(\mathrm{r}) / \mathrm{V}(\mathrm{r})$ ratio between $0.5-1$ ( 0.727 and 0.924 , respectively). For validation and comparison, the related value of BCPs for a $\mathrm{C}-\mathrm{C}$ bond in LHP was measured, and the achieved outcomes showed negative values for $\nabla^{2} \rho$ and $H(r)$, as listed in Table 2. These parameters indicate that exists a covalent bond between carbon atoms in LHP [95]. From the value estimated for $\mathrm{G}(\mathrm{r}) / \mathrm{V}(\mathrm{r}), 0.229$, one can also predict a covalent bond between the neighbor nuclei. 
Table 2

BCPs parameters obtained with revPBE-D3/TZVP model of theory for LHP/Zn cluster system.

\begin{tabular}{|c|c|c|c|}
\hline $\begin{array}{l}\text { BCP1 } \\
(0 . . . Z n)\end{array}$ & $\begin{array}{l}\text { BCP2 } \\
(Z n-Z n)\end{array}$ & $\begin{array}{l}\text { ВСР3 } \\
(C-C)\end{array}$ & \\
\hline$\backslash \operatorname{varvec} \rho(r)$ & 0.053 & 0.049 & 0.255 \\
\hline$\nabla^{2} \backslash \operatorname{varvec} \rho(\mathbf{r})$ & 0.223 & 0.075 & -0.630 \\
\hline$\backslash$ varvec $H(r)$ & -0.005 & -0.013 & -0.225 \\
\hline$G(r)$ & 0.061 & 0.032 & 0.067 \\
\hline$V(r)$ & -0.066 & -0.044 & -0.292 \\
\hline
\end{tabular}

\subsection{DFT-MD simulation of LHP/Zn (110)}

In continue, DFT-MD simulation was implemented to simulate a realistic system comprised of $\mathrm{Zn}$ (110) and LHP inhibitor at environmental conditions. For this objective, the most stable configuration with a stronger interaction energy of the LHP-Zn system (parallel orientation) was considered. Through plotting the total energy of the whole system vs. simulation time step the stability of LHP-Zn was assessed (See Fig. 3a). This plot exhibited that at the initial steps of MD simulation a noticeable decrease in fluctuation occurs at constant values about $-193945.77 \mathrm{eV}$ from $200 \mathrm{fs}$ which exhibits steady stability of the systems after this duration of the simulation. Regarding the outcomes of the DFT-MD simulation, the fluctuation of temperature related to the system occurred at about $300 \mathrm{~K}$. This temperature explains the equilibrium circumstance within simulation time steps (Fig. 3b).

The achieved data indicated that LHP moved toward the Zn surface about $1.58 \AA$ during the simulation procedure (the initial distance between the $\mathrm{Zn}$ surface and LHP molecule was considered about $5.0 \AA$ ), as depicted in Fig. 3c. The observed movement could be further explained through the plots of the distance of $\mathrm{Zn}$... O against the time steps of the simulation. It was found that when LHP has approached in parallel to the $\mathrm{Zn}$ atoms, in the first steps of the simulation, the initial distance of $\mathrm{Zn}$ number 88 ( $\mathrm{nn:88}$ ) with 0 atom number 145 (0:145) was about $5 \AA$ (See Fig. 3d). After 250 fs, this distance notably decreased and reached about $2.2 \AA$.

It was also found that adsorbed LHP diffused on the $\mathrm{Zn}$ surface during the simulation time procedure as demonstrated in Fig. 3e. After LHP adsorption on the Zn surface, an increase in distance of Zn...0 was observed and in $500 \mathrm{fs}$, the distance was about $4.1 \AA$. However, after $500 \mathrm{fs}$ the distance of $\mathrm{Zn}: 88$ decreased to about $2 \AA$, it had an almost stable nuance until $3000 \mathrm{fs}$ and the average distance of $\mathrm{Zn}: 88$ 
with 0 atom was about $2.5 \AA$. At about $3000 \mathrm{fs}$ an incredible increase occurred and the distance of $\mathrm{Zn}$... 0 acceded about $3 \AA$. From the range of $3500-4000 \mathrm{fs}$, the distance was increased and at about $4000 \mathrm{fs}$, the 0:145 atom shifted toward the vicinal Zn:96 (see Fig. 3f). The initial distance of the Zn:96 and 0:145 atoms was about $6 \AA$. After $500 \mathrm{fs}$ the distance decreased to $3.8 \AA$. From the range of $500 \mathrm{fs}$ to $1500 \mathrm{fs}$, the distance increased, and at the time step of $1500-4500 \mathrm{fs}$, the $\mathrm{Zn}$... O distance faced a gradual decline. The final distance of $\mathrm{Zn}$ and $\mathrm{O}$ atoms was found about $2.8 \AA$. The schematic structure of $\mathrm{Zn}$ atoms in the exposure of $\mathrm{O}$ atoms of LHP is shown in Fig. 3d. A similar trend for $\mathrm{Zn:97}$ and 0:139 was surveyed. According to the results of the DFT-MD simulation, when LHP has approached the surface of the $\mathrm{Zn}$ sheet the initial distance of $\mathrm{Zn}$ and $\mathrm{O}$ was about $4.8 \AA$ (Fig. $3 \mathrm{~g}$ ). This distance experienced a remarkable decrease and reached about $2 \AA$ at $480 \mathrm{fs}$. After a slight increase from 500-1000 fs, the distance increased, and it was almost stable until $2800 \mathrm{fs}$. However, it had a sudden decrease in $2000 \mathrm{fs}$. From the range of $2700-4100 \mathrm{fs}$ the distance of $\mathrm{Zn}$... 0 gradually decreased and reached to $4 \AA$. After that, the 0 atom shifted toward Zn:112 (Fig. 3h). The initial distance of $Z n$ and 0 was $6.3 \AA$. Except for two important increases at 1500 and $2500 \mathrm{fs}$, the whole trend of this plot was declining. The final distance of Zn and $\mathrm{O}$ was about $2.8 \AA$.

It should be mentioned that the diffusion of adsorbed molecules on the various metallic surfaces has been observed and reported in detail in the literature [96-100]. For examples, diffusion of large molecules such as acetylene on Pd [96] and Cu [97] surfaces, PVBA on Pd [98], and also aromatic compounds decacyclene (DC) and hexatert-butyl decacyclene (HtBDC) on $\mathrm{Cu}$ [99], azobenzene on Cu [100] surface investigated with obtaining sequences of time-resolved STM.

Furthermore, in Fig. 3k, the plot for the angle between C-C-C (C:135, C:138, and C:142) vs. time step can be seen. According to this plot, it was apperceived that when LHP was located in parallel to $\mathrm{Zn}$ atoms, the initial $\mathrm{C}-\mathrm{C}-\mathrm{C}$ angle was $120^{\circ}$. After $350 \mathrm{fs}$ the angle decreased to $150^{\circ}$ and then, it immediately increased to $130^{\circ}$. From the range of $500-1000 \mathrm{fs}$, a decrease occurred and at $1000 \mathrm{fs}$ the angle of C$\mathrm{C}-\mathrm{C}$ was about $110^{\circ}$. The same increasing and decreasing trend were repeated until $3000 \mathrm{fs}$ and after this time steps, the trend was completely decreasing. At about $4200 \mathrm{fs}$ the angle of $\mathrm{C}-\mathrm{C}-\mathrm{C}$ bonding reached $110^{\circ}$. As a consequence, LHP could be adsorbed strongly on the Zn surface through its 0 active site as well as - $\mathrm{CH}$ groups, though it was diffused on the surface.

\section{Conclusion}

This research was earmarked to the investigation of the behavior of Laurhydrazide N'-propan-3-one (LHP) as an efficient organic inhibitor on Zn (110) sheet by using DFT-MD simulation. In the first step, all considered structures, namely, two parallel layers of Zn sheet and LHP were optimized by the framework of the DFT-D2 method. Two distinct configurations were assigned to assess the interaction of LHP with a metallic substrate. In the first configuration, a molecule of LHP perpendicularly approached the Zn sheet through its $\mathrm{O}$ atom as an active site. In this configuration, the $\mathrm{O}$ atom was able to be located either on one single $\mathrm{Zn}$ atom (top site) or among four $\mathrm{Zn}$ atoms (hollow site). In the second configuration, the LHP molecule was approached in parallel toward the $\mathrm{Zn}$ sheet. Interaction energy for both configurations was 
calculated and it was revealed that when LHP has approached in parallel, creates a more stable configuration with an interaction energy of about $-2.322 \mathrm{eV}$ compared to the first configuration with an energy of about $-1.144 \mathrm{eV}$. The parallel orientation of LHP toward the $\mathrm{Zn}$ sheet was predicted to cover and consequently protect the surface efficiently. In continue, a realistic system comprised of $\mathrm{Zn}(110)$ and the most stable configuration of LHP was simulated using the state-of-the-art DFT-MD method. It was shown that as a result of approaching the LHP, an obvious fluctuation on total energy and bonding distance of $\mathrm{Zn}$... $\mathrm{O}$ was observed during simulation time steps. Further, it was deduced that fluctuation in energy could result in the diffusion of LHP on the surface. In a way that after about $4500 \mathrm{fs}$ of simulation duration, one $\mathrm{O}$ atom shifted from a close neighbor $\mathrm{Zn}$ atom to a further $\mathrm{Zn}$ atom. The same trend was also observed for the second $\mathrm{O}$ atom and adjacent $\mathrm{Zn}$ atoms during the simulation time. In the last part, the calculated adsorption for the most stable configuration was validated by the MP2 level of theory. Our DFT-MD simulation findings meet the goal of the punishment to develop new materials and devices applicable to future coating and corrosion technologies. At the molecular scale, the edge of knowledge between chemistry and physics presented here, provide deep insight into the recent challenges about the interactive nature of inhibitor compounds adsorbed on the metallic surface.

\section{References}

[1] Craddock, P. T. The early history of zinc. Endeavour 11, 183-191 (1987).

[2] Thorne, W. Zinc Deficiency and its Control. Adv. Agron. 9, 31-65 (1957).

[3] Nicholson, J. W. The early history of organotin chemistry. J. Chem. Educ. 66, 621 (1989).

[4] Page, M. A. M., Weidenfeller, B. \& Hartmann, S. Influence of temperature and aging on the thermal diffusivity, thermal conductivity and heat capacity of a zinc die casting alloy. J. Alloys Compd. 786, 10607 (2019).

[5] Page, M. A. M. \& Hartmann, S. Experimental characterization, material modeling, identification and finite element simulation of the thermo-mechanical behavior of a zinc die-casting alloy. Int. J. Plast. 101, 74-105 (2018).

[6] Ou, C, et al. Template-Assisted Hydrothermal Growth of Aligned Zinc Oxide Nanowires for Piezoelectric Energy Harvesting Applications. Appl. Mater. Interfaces 8, 13678-83 (2016).

[7] Bao, S. et al. Highly selective removal of $\mathrm{Zn}(\mathrm{II})$ ion from hot-dip galvanizing pickling waste with aminofunctionalized Fe304@SiO2 magnetic nano-adsorbent. J. Colloid Interface Sci. 462, 235-42 (2016).

[8] Abdollahzadeh, A., Shokuhfar, A., Cabrera, J. M., Zhilyaev, A. P. \& Omidvar, H. The effect of changing chemical composition on dissimilar Mg/Al friction stir welded butt joints using zinc interlayer. J. Manuf. Process. 34, 18-30 (2018). 
[9] Nigam, A. \& Pawar, S. J. Structural, magnetic, and antimicrobial properties of zinc doped magnesium ferrite for drug delivery applications. Ceram. Int. 46, 4058-64 (2020).

[10] Almeida, C. M. V. B. et al. Accounting for the benefits of technology change: Replacing a zinc-coating process by a water-based organo-metallic coating process. J. Clean. Prod. 174, 170-6 (2018).

[11] Hernández-escobar, D. et al. Current status and perspectives of zinc-based absorbable alloys for biomedical applications. Acta Biomater. 97, 1-22 (2019).

[12] Sumboja, A. et al. Durable rechargeable zinc-air batteries with neutral electrolyte and manganese oxide catalyst. J. Power Sources 332, 330-6 (2016).

[13] Tan, P. et al. In-situ growth of Co3O4 nanowire-assembled clusters on nickel foam for aqueous rechargeable Zn-Co304 and Zn-air batteries. Applied Catal. B, Environ. 241, 104-12 (2019).

[14] Yan, X. et al. Self-assembled Zn-Al-F layered double hydroxides enabling dendrite-free Zn deposition process in high-rate Ni-Zn secondary batteries. J. Power Sources 448, 227412 (2020).

[15] Ma, M. et al. Electrochemical performance of $\mathrm{ZnO}$ nanoplates as anode materials for $\mathrm{Ni} / \mathrm{Zn}$ secondary batteries. J. Power Sources 179, 395-400 (2008).

[16] Zheng, Y. et al. Effects of barium on the performance of secondary alkaline zinc electrode. Mater. Chem. Phys. 84, 99-106 (2004).

[17] Tuken, T., Yazıcı, B. \& Erbil, M. Mater. Zinc modified polyaniline coating for mild steel protection. Chem. Phys. 99, 459-64 (2006).

[18] Hayatdavoudi, H. \& Rahsepar, M. A mechanistic study of the enhanced cathodic protection performance of graphene-reinforced zinc rich nanocomposite coating for corrosion protection of carbon steel substrate. J. Alloys Compd. 727, 1148-56 (2017).

[19] Abrishami, S., Naderi, R. \& Ramezanzadeh, B. Fabrication and characterization of zinc acetylacetonate/Urtica Dioica leaves extract complex as an effective organic/inorganic hybrid corrosion inhibitive pigment for mild steel protection in chloride solution. Appl. Surf. Sci. 457, 487-96 (2018).

[20] Xu, R., He, T., Yang, R., Da, Y. \& Chen, C. Application zinc silicate-potassium silicate coating for anticorrosion of steel bar in autoclaved aerated concrete. Constr. Build. Mater. 237, 117521 (2020).

[21] Xie, Y., Chen, M., Xie, D., Zhong, L. \& Zhang, X. A fast, low temperature zinc phosphate coating on steel accelerated by graphene oxide. Corros. Sci. 128, 1-8 (2017).

[22] Jo, Y. N., Kang, S. H., Prasanna, K., Eom, S. W. \& Lee, C. W. Shield effect of polyaniline between zinc active material and aqueous electrolyte in zinc-air batteries. Appl. Surf. Sci. 422, 406-12 (2017). 
[23] Das, M. et al. Enhanced pseudo-halide promoted corrosion inhibition by biologically active zinc(II) Schiff base complexes. Chem. Eng. J. 357, 447-57 (2019).

[24] Wittman, R. M., Sacci, R. L. \& Zawodzinski, T. A. Elucidating mechanisms of oxide growth and surface passivation on zinc thin film electrodes in alkaline solutions using the electrochemical quartz crystal microbalance. J. Power Sources. 438, 227034 (2019).

[25] Liu, Y., Ooi, A., Tada, E. \& Nishikata, A. Electrochemical monitoring of the degradation of galvanized steel in simulated marine atmosphere. Corros. Sci. 147, 273-82 (2019).

[26] Wang, P., Zhang, D., Qiu, R., Wu, J. \& Wan, Y. Super-hydrophobic film prepared on zinc and its effect on corrosion in simulated marine atmosphere. Corros. Sci. 69, 23-30 (2013).

[27] Kakaei, M. N., Danaee, I. \& Zaarei, D. Investigation of corrosion protection afforded by inorganic anticorrosive coatings comprising micaceous iron oxide and zinc dust. Corros. Eng. Sci. Technol. 48, 194-8 (2013).

[28] Jurak, T. et al. Novel Chromium-Free Technologies for the Prevention of Wet Stack Corrosion on Hot Dipped Metallic Coatings: A Review. Mater. Corros. 66, 1051-9 (2015).

[29] Verma, C., Olasunkanmi, L. O., Obot, I. B., Ebenso, E. E. \& Quraishi, M. A. 2,4-Diamino-5(phenylthio)-5H-chromeno [2,3-b] pyridine-3-carbonitriles as green and effective corrosion inhibitors: gravimetric, electrochemical, surface morphology and theoretical studies. RSC Adv. 6, 53933-48 (2016).

[30] Nmai, C. K. Multi-functional organic corrosion inhibitor, Cem. Concr. Compos. 26, 199-207 (2004).

[31] El-Hajjaji, F. et al. Effect of 1-(3-phenoxypropyl) pyridazin-1-ium bromide on steel corrosion inhibition in acidic medium. J. Colloid Interface Sci. 541, 418-24 (2019).

[32] Galai, M. et al. New Hexa Propylene Glycol Cyclotiphosphazene as Efficient Organic Inhibitor of Carbon Steel Corrosion in Hydrochloric Acid Medium. J. Mater. Environ. Sci. 7, 1562-75 (2016).

[33] Dagdag, O. et al. Anticorrosive properties of Hexa (3-methoxy propan-1,2-diol) cyclotri-phosphazene compound for carbon steel in $3 \% \mathrm{NaCl}$ medium: gravimetric, electrochemical, DFT and Monte Carlo simulation studies. Heliyon 5, e01340 (2019).

[34] Hsissou, R. et al. Experimental, DFT and molecular dynamics simulation on the inhibition performance of the DGDCBA epoxy polymer against the corrosion of the E24 carbon steel in $1.0 \mathrm{M} \mathrm{HCl}$ solution. J. Mol. Struct. 1182, 340-51 (2019).

[35] Goyal, M., Kumar, S., Bahadur, I., Verma, C. \& Ebenso, E. E. Organic corrosion inhibitors for industrial cleaning of ferrous and non-ferrous metals in acidic solutions: A review. J. Mol. Liq. 256, 565-73 (2018). 
[36] Ju, H., Kai, Z. \& Li, Y. Aminic nitrogen-bearing polydentate Schiff base compounds as corrosion inhibitors for iron in acidic media: A quantum chemical calculation. Corros. Sci. 50, 865-71 (2008).

[37] Boughoues, Y., Benamira, M., Messaadia, L. \& Ribouh, N. Adsorption and corrosion inhibition performance of some environmental friendly organic inhibitors for mild steel in $\mathrm{HCl}$ solution via experimental and theoretical study. Colloids Surfaces A Physicochem. Eng. Asp. 593, 124610 (2020).

[38] Zheng, S. \& Li, J. Inorganic-organic sol gel hybrid coatings for corrosion protection of metals. J. SolGel Sci. Technol. 54, 174-87 (2010).

[39] Kovacevic, N. \& Kokalj, A. Chemistry of the interaction between azole type corrosion inhibitor molecules and metal surfaces. Mater. Chem. Phys. 137, 331-9 (2012).

[40] Schreiner, P.R. Metal-free organocatalysis through explicit hydrogen bonding interactions. Chem. Soc. Rev. 32, 289-96 (2003).

[41] Anupama, K.K., Ramya, K. \& Joseph, A. Electrochemical and computational aspects of surface interaction and corrosion inhibition of mild steel in hydrochloric acid by Phyllanthus amarus leaf extract (PAE). J. Mol. Liq. 216, 146-55 (2016).

[42] Ormellese, M., Lazzari, L., Goidanich, S., Fumagalli, G. \& Brenna, A. A study of organic substances as inhibitors for chloride-induced corrosion in concrete. Corros. Sci. 51, 2959-68 (2009).

[43] Guo, L. et al. Theoretical insight into an empirical rule about organic corrosion inhibitors containing nitrogen, oxygen, and sulfur atoms. Appl. Surf. Sci. 406, 301-6 (2017).

[44] Yadav, M., Sarkar, T. K. \& Purkait, T. Amino acid compounds as eco-friendly corrosion inhibitor for N80 steel in $\mathrm{HCl}$ solution: Electrochemical and theoretical approaches. J. Mol. Liq. 212, 731-8 (2015).

[45] Simonovic, A. T., Petrovic, M. B., Radovanovic, M. B., Milic, S. M. \& Antonijevic, M. M. Inhibition of copper corrosion in acidic sulphate media by eco-friendly amino acid compound. Chem. Pap. 68, 362-71 (2014).

[46] Zhang, D., Gao, L. \& Zhou, G. Inhibition of copper corrosion in aerated hydrochloric acid solution by amino-acid compounds. J. Appl. Electrochem. 35, 1081-5 (2005).

[47] Zhang, T., Jiang, W., Wang, H. \& Zhang, S. Synthesis and localized inhibition behaviour of new triazine-methionine corrosion inhibitor in $1 \mathrm{M} \mathrm{HCl}$ for 2024-T3 aluminium alloy. Mater. Chem. Phys. 237, 121866 (2019).

[48] Goni, L. K. M. O., Mazumder, M. A. J., Ali, S. A. \& Nazal, M. K. Biogenic amino acid methionine-based corrosion inhibitors of mild steel in acidic media. Int. J. Miner. Metall. Mater. 26, 467-82 (2019). 
[49] Li, B. et al. Automated inference of molecular mechanisms of disease from amino acid substitutions. Bioinformatics 25, 2744-2750 (2009).

[50] Shang, P. et al. The amino acid transporter SLC36A4 regulates the amino acid pool in retinal pigmented epithelial cells and mediates the mechanistic target of rapamycin, complex 1 signaling. Aging Cel/ 16, 349-59 (2017).

[51] Mitchell, W. K. et al. Human Skeletal Muscle Protein Metabolism Responses to Amino Acid Nutrition Adv. Nutr. 7, 828S-838S (2016).

[52] Taylor, W. R. The classification of amino acid conservation. J. theor. Biol. 119, 205-18 (1986).

[53] Tabrez, M., Shamim, A., Anwaruddin, M. \& Nagarajaram, H. A. Support Vector Machine-based classification of protein folds using the structural properties of amino acid residues and amino acid residue pairs. Bioinformatics 23,3320-7 (2007).

[54] Campbell, J. A., Davies, G. J., Bulone, V. \& Henrissat, B. A classification of nucleotide-diphospho-sugar glycosyltransferases based on amino acid sequence similarities. Biochem J. 326, 929-39 (1997).

[55] Zhang, D. Q., Cai, Q. R., Gao, L-X. \& Yong, K. Y. Effect of serine, threonine and glutamic acid on the corrosion of copper in aerated hydrochloric acid solution. Corros. Sci. 50, 3615-21 (2008).

[56] Fu, J., Li, S., Wang, Y., Cao, L. \& Lu, L. Computational and electrochemical studies of some amino acid compounds as corrosion inhibitors for mild steel in hydrochloric acid solution. J. Mater. Sci. 45, 6255-65 (2010).

[57] El-hafez, G. M. A. \& Badawy, W. A. The use of cysteine, N-acetyl cysteine and methionine as environmentally friendly corrosion inhibitors for $\mathrm{Cu}-10 \mathrm{Al}-5 \mathrm{Ni}$ alloy in neutral chloride solutions. Electrochim. Acta. 108, 860-66 (2013).

[58] Badawy, W. A., Ismail, K. M. \& Fathi, A. M. Corrosion control of $\mathrm{Cu}-\mathrm{Ni}$ alloys in neutral chloride solutions by amino acids. Electrochim. Acta 51, 4182-9 (2006).

[59] Ashassi-sorkhabi, H., Majidi, M. R. \& Seyyedi, K. Investigation of inhibition effect of some amino acids against steel corrosion in $\mathrm{HCl}$ solution. Appl. Surf. Sci. 225, 76-185 (2004).

[60] Kaya, S., Tuzun, B., Kaya, C. \& Obot, I. B. Determination of corrosion inhibition effects of amino acids: Quantum chemical and molecular dynamic simulation study. J. Taiwan Inst. Chem. Eng. 58, 528-35 (2016).

[61] El-haddad., M. A. M., Radwan, A. B., Sliem, M. H., Hassan, W. M. I. \& Abdullah, A. M. Highly efficient eco-friendly corrosion inhibitor for mild steel in $5 \mathrm{M} \mathrm{HCl}$ at elevated temperatures: experimental \& molecular dynamics study. Sci. Rep. 9, 3695 (2019). 
[62] Soler, J. M. et al. The SIESTA method for ab initio Order-N materials simulation. J. Phys.: Condens. Matter 14, 2745 (2002).

[63] Artacho, E. et al. The SIESTA method; developments and applicability J. Phys.: Condens. Matter 20, 1 (2008).

[64] Perdew, J. P., Burke, K. \& Ernzerhof, M. Generalized Gradient Approximation Made Simple. Phys. Rev. Lett. 77, 3865 (1996).

[65] Zhang, Y. \& Yang, W. Comment on "Generalized Gradient Approximation Made Simple". Phys. Rev. Lett. 80, 890 (1998).

[66] Hammer, B., Hansen, L. B. \& Nørskov, J. K. Improved adsorption energetics within density-functional theory using revised Perdew-Burke-Ernzerhof functionals. Phys. Rev. B 59, 7413-21 (1999).

[67] Staroverov, V. N., Scuseria, G. E., Perdew, J. P., Davidson E. R. \& Katriel J. High-density limit of the Perdew-Burke-Ernzerhof generalized gradient approximation and related density functionals. J. Phys. Rev. A 74, 044501 (2006).

[68] Troullier, N. \& Martins, J. L. Efficient pseudopotentials for plane-wave calculations. Phys. Rev. B 43, 1991, 1993-2006.

[69] Soler, J. M. et al. The SIESTA method for ab initio Order-N materials simulation. J. Phys. Condens. Matter 14, 2745-79 (2002).

[70] Ozaki, H. K. T., Yu, J., Han, M. J., Kobayashi, N., Ohfuti, M., Ishii, F., Ohwaki, T. \& Weng, H. Available: http://www.openmx-square.org/.

[71] Morrison, I., Bylander, D. M. \& Kleinman, L. Nonlocal Hermitian norm-conserving Vanderbilt pseudopotential. Phys. Rev. B 47, 6728 (1993).

[72] Grimme, S. Semiempirical GGA-type density functional constructed with a long-range dispersion correction. J. Comput. Chem. 27, 1787-99 (2006).

[73] Boys, S. F. \& Bernardi, F. d. The calculation of small molecular interactions by the differences of separate total energies. Some procedures with reduced errors. Mol. Phys. 19, 553-66 (1970).

[74] Schäfer, A., Huber, C. \& Ahlrichs, R. Fully optimized contracted Gaussian basis sets of triple zeta valence quality for atoms Li to Kr. J. Chem. Phys. 100, 5829-35 (1994).

[75] Haeussermann, U., Dolg, M., Stoll, H. \& Preuss, H. Accuracy of energy-adjusted quasirelativistic ab initio pseudopotentials. Mol. Phys. 78, 1211-24 (1993).

[76] Peterson, K. A., Figgen, D., Goll, E. \& Stoll, H. Systematically convergent basis sets with relativistic pseudopotentials. II. Small-core pseudopotentials and correlation consistent basis sets for the post-d 
group 16-18 elements. J. Chem. Phys. 119, 11113-23 (2003).

[77] Bader, R. F. W. Comment on: Revisiting the variational nature of the quantum theory of atoms in molecules. Chem. Phys. Lett. 426, 226-8 (2006).

[78] Popelier, P. L., Simos, T. E. \& Wilson, S. Chemical Modelling: Applications and Theory. Vol. 1. (Royal Society of Chemistry, 2000).

[79] Cortés-Guzmán, F. \& Bader, R.F. W. Complementarity of QTAIM and MO theory in the study of bonding in donor-acceptor complexes. Coord. Chem. Rev. 249, 633-62 (2005).

[80] Lu, T. \& Chen F. J. Multiwfn: A multifunctional wavefunction analyzer. Comput. Chem. 33, 580-592 (2012).

[81] Kundrat, M. D. \& Autschbach, J. Time Dependent Density Functional Theory Modeling of Specific Rotation and Optical Rotatory Dispersion of the Aromatic Amino Acids in Solution. J. Phys. Chem. A 110, 12908-17 (2006).

[82] Arefian, M., Mirzaei, M. \& Eshtiagh-hosseini, H. Structural insights into two inorganic-organic hybrids based on chiral amino acids and polyoxomolybdates. J. Mol. Struct. 1156, 550-8 (2018).

[83] Kundrat, M. D. \& Autschbach, J. Modeling of the Chiroptical Response of Chiral Amino Acids in Solution Using Explicit Solvation and Molecular Dynamics. J. Chem. Theory Comput. 5, 1051-60 (2009).

[84] Chowdhry, B. Z., Dines, T. J., Jabeen, S. \& Withnall, R. Vibrational Spectra of a-Amino Acids in the Zwitterionic State in Aqueous Solution and the Solid State: DFT Calculations and the Influence of Hydrogen Bonding. J. Phys. Chem. A 112, 10333-47 (2008).

[85] Xu, W. Z. et al. Quasi-aligned ZnO nanotubes grown on Si substrates. Appl. Phys. Lett. 87, 093110 (2005).

[86] Xing, Y. J., Xi, Z. H., Xue, Z. Q., Zhang, X. D. \& Song, J. H. Optical properties of the ZnO nanotubes synthesized via vapor phase growth. Appl. Phys. Lett. 83, 1689-91 (2003).

[87] Wei, A. et al. Stable field emission from hydrothermally grown ZnO nanotubes. Appl. Phys. Lett. 88, 213102-5 (2006).

[88] Xu, C. X. et al. Growth and spectral analysis of ZnO nanotubes. Appl. Phys. 103, 094303 (2008).

[89] Ju, H. \& Li, Y. Nicotinic acid as a nontoxic corrosion inhibitor for hot dipped Zn and Zn-Al alloy coatings on steels in diluted hydrochloric acid. Corros. Sci. 49, 4185-201 (2007).

[90] Izakmehri, Z., Ardjmand, M., Ganji, M. D., Babanezhad, E. \& Heydarinasab, A. Removal of dioxane pollutants from water by using Al-doped single walled carbon nanotubes. RSC Advances 5, 48124-48132 (2015). 
[91] Soleymani, E., Alinezhad, H., Ganji, M. D. \& Tajbakhsh, M. Enantioseparation performance of CNTs as chiral selectors for the separation of ibuprofen isomers: a dispersion corrected DFT study. Journal of Materials Chemistry B 5, 6920-6929 (2017).

[92] Larijani, H. T., Jahanshahi, M., Ganji, M. D. \& Kiani, M. Computational studies on the interactions of glycine amino acid with graphene, h-BN and h-SiC monolayers. Physical Chemistry Chemical Physics 19, 1896-1908 (2017).

[93] Alinezhad, H., Ganji, M. D., Soleymani, E. \& Tajbakhsh, M. A comprehensive theoretical investigation about the bio-functionalization capability of single walled CNT, BNNT and SiCNT using DNA/RNA nucleobases. Applied Surface Science 422, 56-72 (2017).

[94] Ganji, M. D., Larijani, H. T., Alamol-Hoda, R. \& Mehdizadeh, M. First-principles and Molecular Dynamics simulation studies of functionalization of Au 32 golden fullerene with amino acids. Scientific reports 8, 1-13 (2018).

[95] Sjoberg, P., Murray, J. S., Brinck, T. \& Politzer, P. Average local ionization energies on the molecular surfaces of aromatic systems as guides to chemical reactivity. Can. J. Chem. 68, 1440-1443 (1990).

[96] Dunphy, J. C. et al. Acetylene structure and dynamics on Pd (111). Phys. Rev. B 57, R12705-8 (1998).

[97] Lauhon, L. J. \& Ho, W. Single molecule thermal rotation and diffusion: Acetylene on Cu (001). J. Chem. Phys. 111, 5633-6 (1999).

[98] Weckesser, J., Barth, J. V. \& Kern, K. Direct observation of surface diffusion of large organic molecules at metal surfaces: PVBA on Pd (110). J. Chem. Phys. 110, 5351-4 (1999).

[99] Schunack, M. et al. Long Jumps in the Surface Diffusion of Large Molecules. Phys. Rev. Lett. 88, 156102 (2002).

[100] Miwa, J. A. et al. Azobenzene on Cu (110): Adsorption Site-Dependent Diffusion. J. Am. Chem. Soc. $128,3164-5(2006)$.

\section{Figures}


(a)

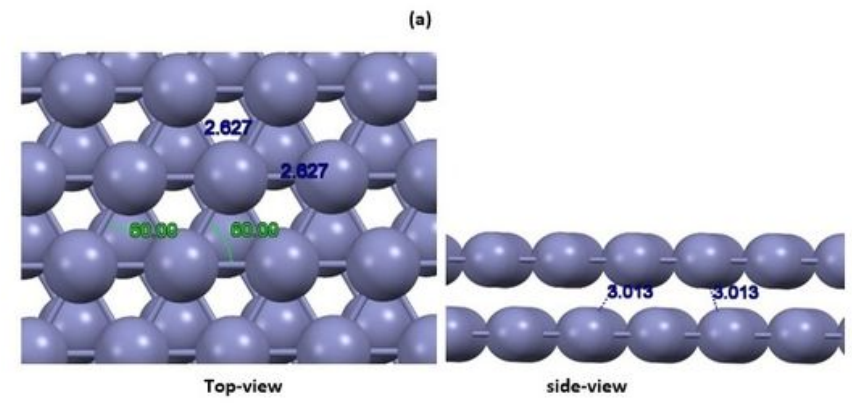

(b)

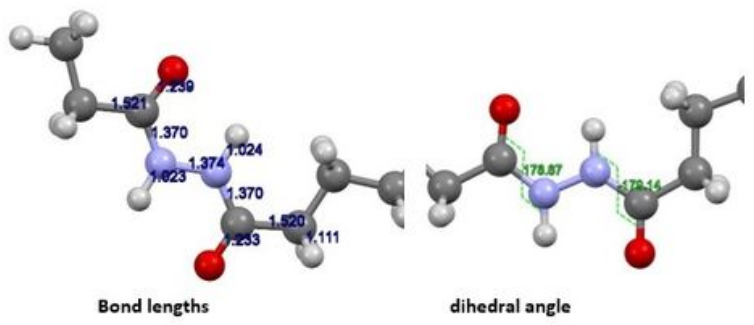

(c)

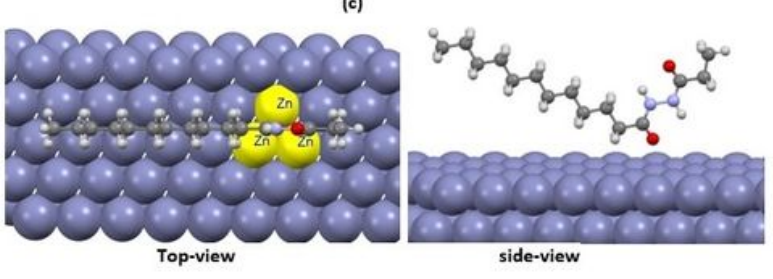

(d)

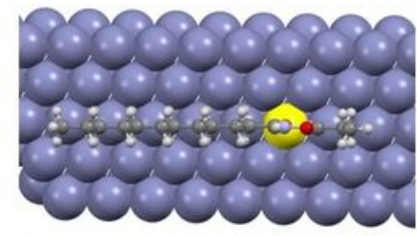

Top-view

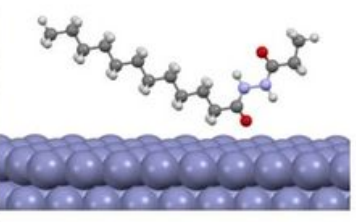

side-view

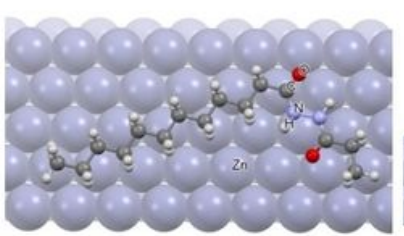

.

(19.6.9.8.

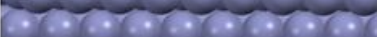

\section{Figure 1}

Bonding distances and angels of vicinal atoms for DFT optimized of (a) Zn sheet and (b) LHP molecule. Initial configurations for approaching LHP on the $\mathrm{Zn}$ surface through its $\mathrm{O} / \mathrm{H}$ atoms (c) perpendicularhollow, (d) perpendicular-top, and (e) parallel orientations. 
(a)
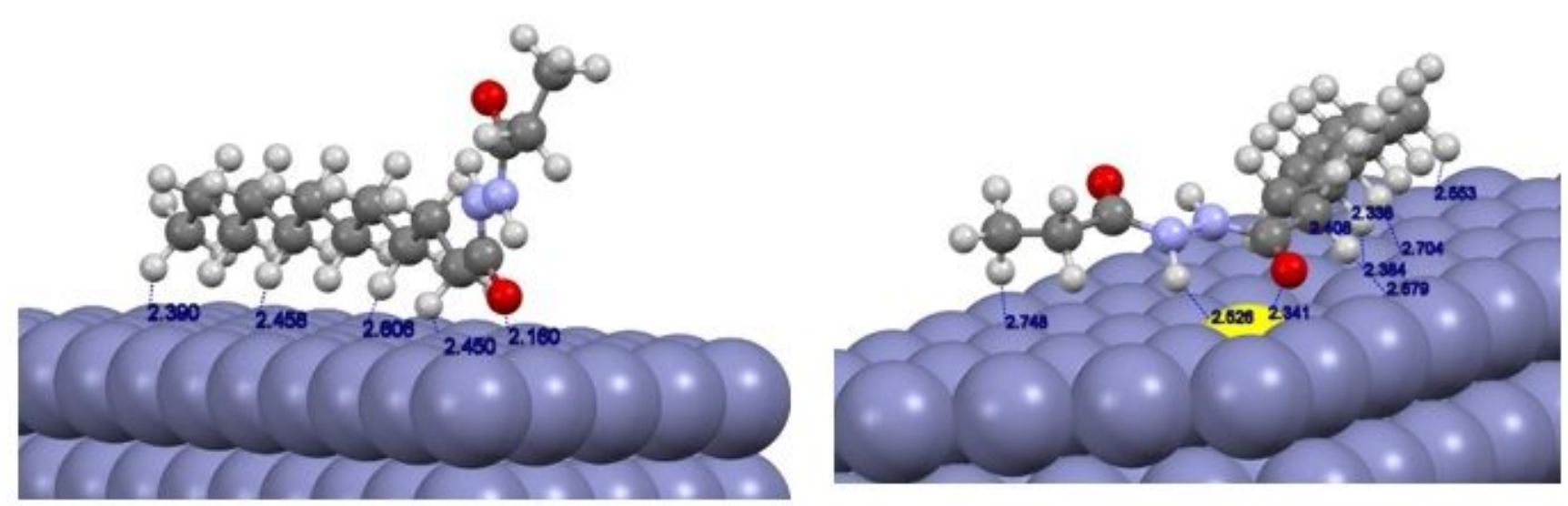

(c)

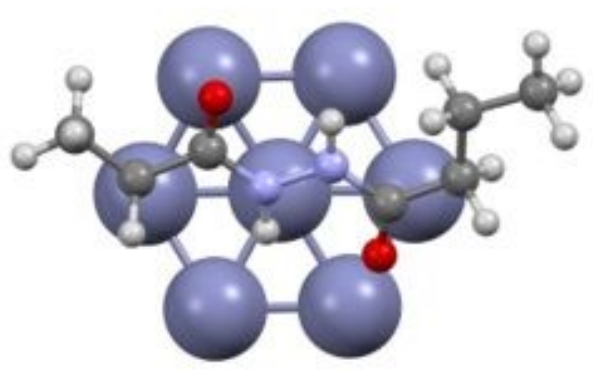

Top-view (b)

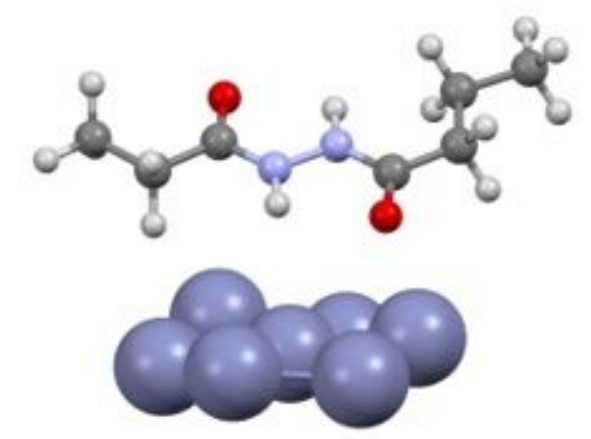

Side-view

(e)
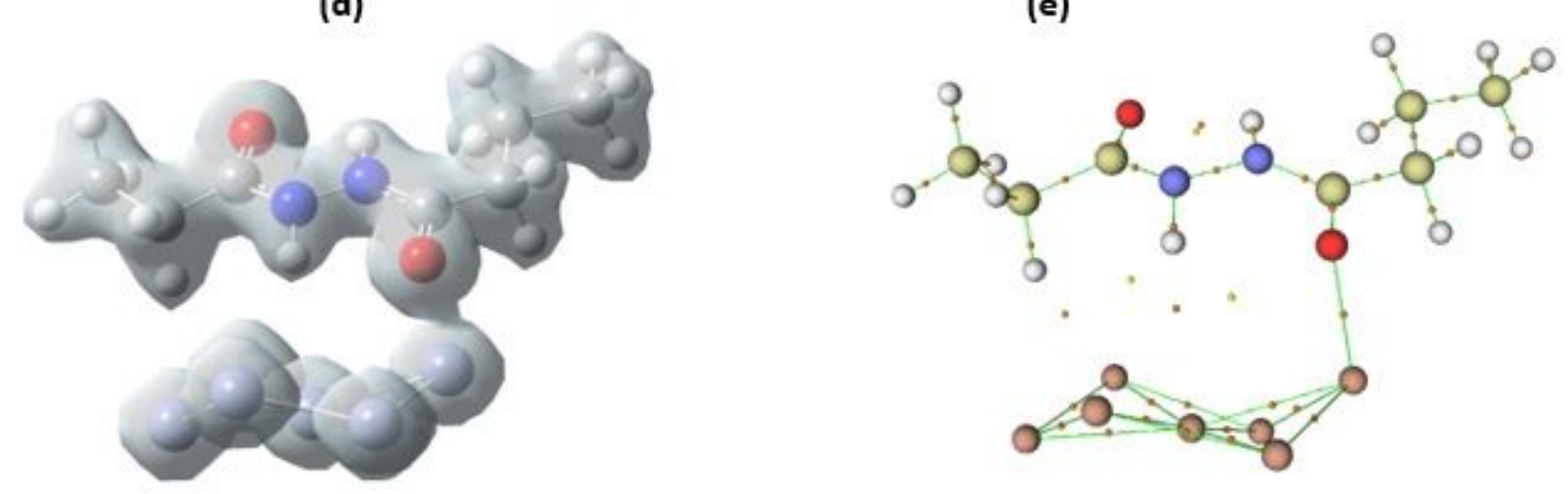

\section{Figure 2}

Equilibrium distances between $\mathrm{O} / \mathrm{H}$ atom and $\mathrm{Zn}$ surface in optimized LHP/Zn system for (a) energetically favorable perpendicular (top) and (b) parallel orientation. Representation of (c) cluster model for LHP/Zn7 system, (d) calculated total charge density of LHP/Zn7 with PBE-D3/TZVP (isovalue was set to 0.07 a.u.). (e) Calculated bonding critical points (BCPs) with PBE-D3/TZVP for LHP/Zn7 system. 

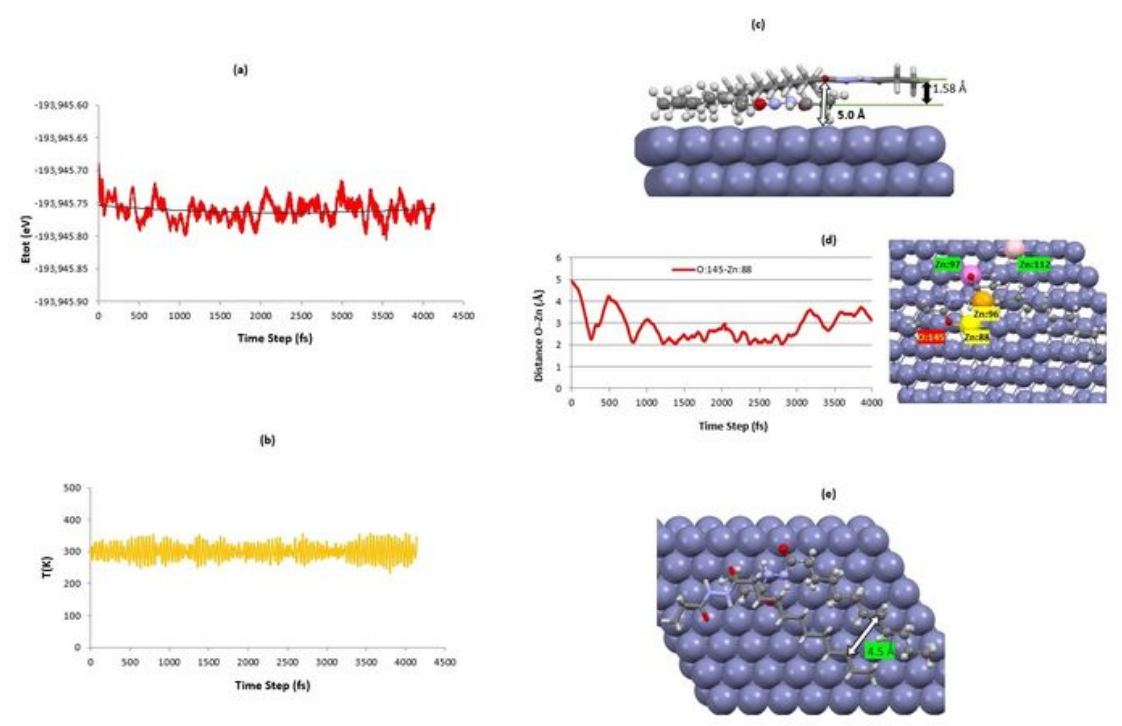

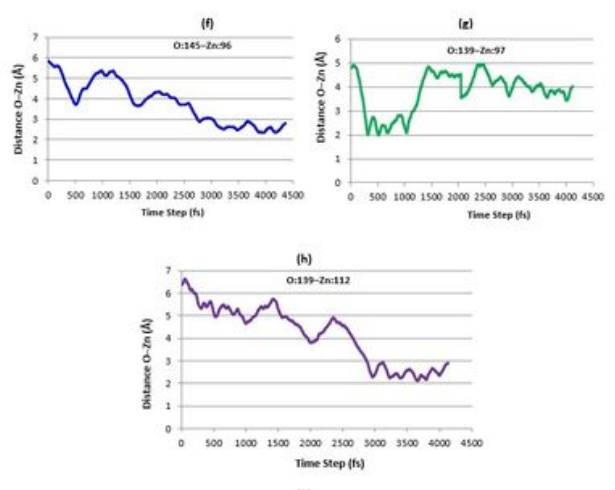

(4)

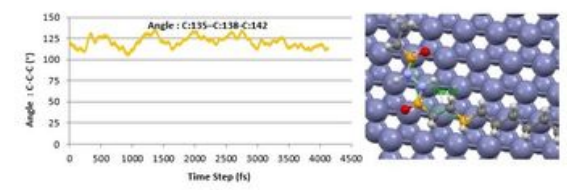

\section{Figure 3}

Plots of (a) total energy and (b) temperature $(K)$ vs. simulation time steps for parallel configuration of LHP/Zn(110) system. (c) Representation of movement of LHP during the simulation procedure. (d) The plot of the bonding distance between 0:145 and Zn:88 during the simulation time (atoms index is shown on the right-hand side). (e) Schematic depiction of diffused LHP molecule on the $\mathrm{Zn}$ surface during the simulation procedure. Plots of bond distances of (f) 0:145 and Zn:96, (g) 0:139 and Zn:97, (h) 0:139 and $\mathrm{Zn:112}$ and (k) C:135-C:138-C:142 during the simulation times. 